\title{
Computer Simulation of Anterograde Accessory Pathway Conduction in Wolff-Parkinson-White Syndrome with a Simplified Model
}

\author{
Ryo Haraguchi $^{1}$, Takashi Ashihara ${ }^{2}$, Taka-aki Matsuyama ${ }^{3}$, Jun Yoshimoto ${ }^{4}$ \\ ${ }^{1}$ Graduate School of Applied Informatics, University of Hyogo, Kobe, Japan \\ ${ }^{2}$ Shiga University of Medical Science, Otsu, Japan \\ ${ }^{3}$ Showa University, Tokyo, Japan \\ ${ }^{4}$ Shizuoka Children's Hospital, Shizuoka, Japan
}

\begin{abstract}
The mechanism underlying Wolff-Parkinson-White syndrome features accessory conduction between the atrium and ventricle. However, the morphological and electrophysiological details remain unclear. Here, we performed preliminary computer simulations of anterograde, accessory pathway conduction using a simplified wall model. Our model features simplified atrial and ventricular walls, and a myocardial bundle (the accessory pathway). The action potentials of these components were represented by Courtemenche and O'Hara-Rudy models. We performed computer simulations to explore relationships among accessory pathway bundle size, intercellular conductivity, and anterograde conduction. We found that a thick accessory pathway facilitates anterograde conduction, ventricular conductivity promotes such conduction, and the accessory pathway prevents anterograde conduction. Sourcesink mismatch may explain the first two points; an electrotonic effect may explain the last one. Our findings provide new insights into the morphological and electrophysiological details of the accessory pathway.
\end{abstract}

\section{Introduction}

Wolff-Parkinson-White (WPW) syndrome is a congenital heart conduction disease involving the accessory pathways (Aps), which leads to the development of cardiac arrhythmias[1]. The underlying mechanism involves an AP composed of ordinary working myocardium connecting the atrium to ventricle[2]. Clinically, radiofrequency AP catheter ablation is favored for treatment[3-5]. However, the morphological and electrophysiological details of the condition remain unclear.

We recently used three-dimensional image reconstruction to visualize the histological morphology of the bundle of Kent (an AP)[6]. Wei et al. reported that electrophysiological WPW syndrome can be successfully simulated on a computer[7], but there are few reports on AP morphology/electrophysiology[8,9].

It is widely accepted that a narrow AP poorly sustains electrical conduction[10]. Myocardial electrical conductivity affects conductive sustainment. We performed preliminary computer simulations of AP conduction using a simplified three-dimensional (3D) wall model to identify the morphological and electrophysiological characteristics of AP. We focused on AP bundle size and myocardial conductivity.

\section{Methods}

We constructed a simplified 3D model featuring atrial and ventricular walls, and a myocardial bundle (Fig. 1). The atrial model combines two walls $20 \times 15 \times 1.05 \mathrm{~mm}$ and $20 \times 10 \times 1.05 \mathrm{~mm}$ in dimension. The ventricular wall dimensions are $20 \times 25 \times 7.05 \mathrm{~mm}$. The atrial and ventricular models featured 145,103 and 1,034,208 discrete myocardial units, respectively. AP models varying in terms of bundle size were integrated with the atrial and ventricular models, for a total of five models (Table 1).
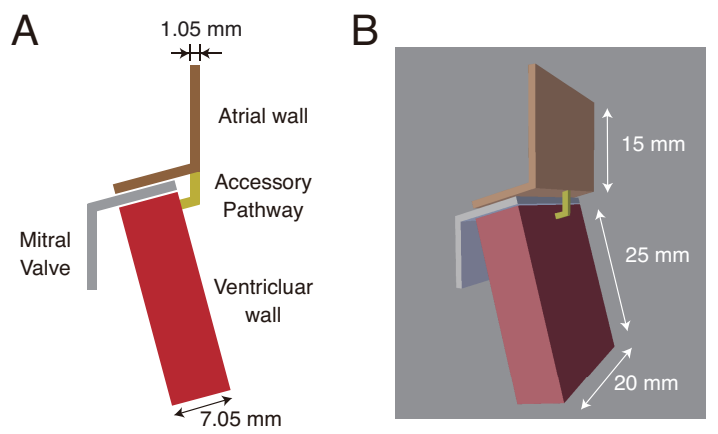

Figure 1. The three-dimensional simplified model. (A) cross-sectional view; (B) three-dimensional view 
Table 1. Configurations of the accessory pathway models.

\begin{tabular}{ccc}
\hline \hline Name & $\begin{array}{c}\text { Accessory pathway's } \\
\text { section size }\end{array}$ & $\begin{array}{c}\text { Number of units } \\
\text { in accessory pathway }\end{array}$ \\
\hline model A & $0.60 \times 0.60 \mathrm{~mm}$ & 524 \\
model B & $0.75 \times 0.75 \mathrm{~mm}$ & 825 \\
model C & $0.90 \times 0.90 \mathrm{~mm}$ & 1,044 \\
model D & $1.05 \times 1.05 \mathrm{~mm}$ & 1,610 \\
model E & $1.95 \times 1.05 \mathrm{~mm}$ & 2,990 \\
\hline \hline
\end{tabular}

The membrane kinetics of the atrial wall and AP were produced by Courtemanche mathematical equations[11]. The membrane kinetics of the ventricular wall was produced by O'Hara-Rudy model equations[12] with modification of the conductance of the sodium channel current. The model was configured as a monodomain, and the forward Euler method was adopted for the integral calculation[13]. The tissue border was set to no-flux Neumann boundary condition. Isotropic intercellular conductivity was configured to ensure that the conduction velocity lay in the physiological range of the experimental data[14,15]. Figure 2 shows the relationship between intercellular conductance and conduction velocity.

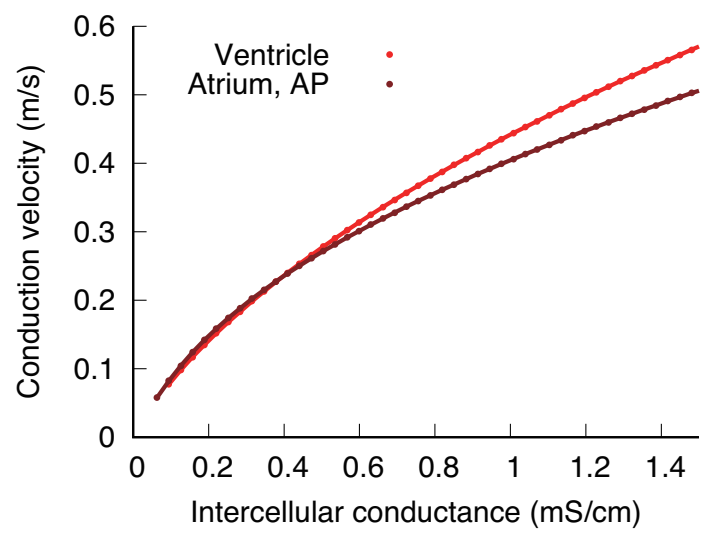

Figure 2. Relationship between intercellular conductance and conduction velocity.

Assuming anterograde (thus atrium-to-ventricle) conduction, excitations were propagated by delivering 10 pacing stimuli to the top of the atrial wall (cycle length: $1,000 \mathrm{~ms}$ ). If anterograde conduction via an AP was in play, these excitations would be propagated within the ventricle. We set the simulation time to $11,000 \mathrm{~ms}$ after initial pacing to optimize computational performance.

\section{Results}

\subsection{A thick accessory pathway triggers an- terograde conduction}

First, we set the overall isotropic intercellular conductance to $0.17 \mathrm{mS} / \mathrm{cm}$. As shown in Figure 3, the excitation propagation ran from the atria to the AP, but not from the AP to the ventricle, in model C. By contrast, in model $\mathrm{D}$, excitation propagation ran from the atria to the ventricle via the AP (model D featured a thicker atrial bundle). Table 2 summarizes the relationship between accessory pathway bundle size and anterograde conduction. A thick accessory pathway triggered such conduction. In other words, a small bundle blocked conduction.

\section{A Conduction block (model C)}

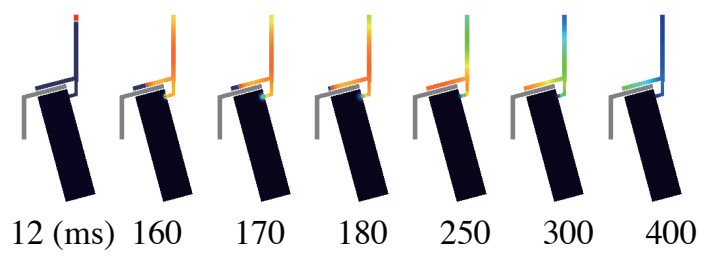

B Anterograde conduction (model D)

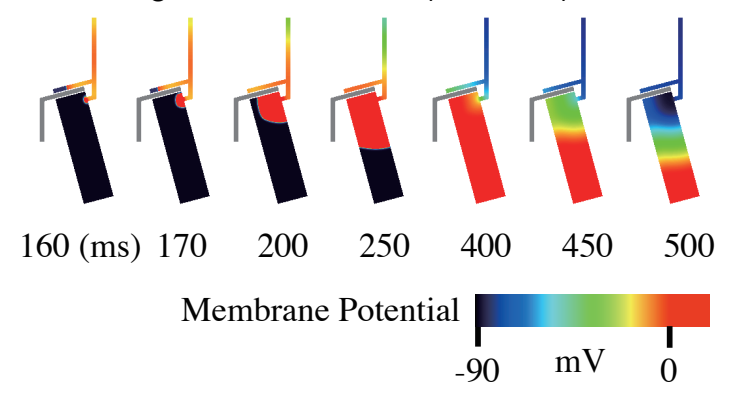

Figure 3. The snapshots of anterograde conduction

Table 2. Relationship between the cross-section of the accessory pathway and anterograde conduction.

\begin{tabular}{ccc}
\hline \hline Model name & $\begin{array}{c}\text { Accessory pathway's } \\
\text { section size }\end{array}$ & $\begin{array}{c}\text { Anterograde } \\
\text { conduction }\end{array}$ \\
\hline model A & $0.60 \times 0.60 \mathrm{~mm}$ & block \\
model B & $0.75 \times 0.75 \mathrm{~mm}$ & block \\
model C & $0.90 \times 0.90 \mathrm{~mm}$ & block \\
model D & $1.05 \times 1.05 \mathrm{~mm}$ & success \\
model E & $1.95 \times 1.05 \mathrm{~mm}$ & success \\
\hline \hline
\end{tabular}




\subsection{Ventricular conductivity promotes an- terograde conduction}

Next, we increased ventricular conductivity within its physiological range. Table 3 shows the relationship between such conductivity and anterograde conduction afforded by model C. Ventricular wall conductivity promoted anterograde conduction.

Table 3. Relationship between ventricular conductivity and anterograde conduction in model $\mathrm{C}$.

\begin{tabular}{cc}
\hline \hline $\begin{array}{c}\text { Ventricular conductivity } \\
(\mathrm{mS} / \mathrm{cm})\end{array}$ & $\begin{array}{c}\text { Anterograde } \\
\text { conduction }\end{array}$ \\
\hline 0.17 & block \\
0.20 & success \\
0.40 & success \\
0.60 & success \\
0.79 & success \\
1.36 & success \\
\hline \hline
\end{tabular}

\subsection{AP conductivity prevents anterograde conduction}

Next, we increased AP conductivity. Table 4 shows the relationship between such conductivity and the anterograde conduction of model D. AP conductivity prevented anterograde conduction.

Table 4. Relationship between AP conductivity and anterograde conduction in model D.

\begin{tabular}{cc}
\hline \hline $\begin{array}{c}\text { AP conductivity } \\
(\mathrm{mS} / \mathrm{cm})\end{array}$ & $\begin{array}{c}\text { Anterograde } \\
\text { conduction }\end{array}$ \\
\hline 0.17 & success \\
0.20 & success \\
0.40 & success \\
0.60 & success \\
0.79 & block \\
1.36 & block \\
\hline \hline
\end{tabular}

\section{Discussion}

We explored the relationship among AP bundle size, intercellular conductivity, and anterograde conduction in WPW syndrome. We investigated whether excitation propagation ran from the atria to the ventricle via an AP. When bundle size increased, anterograde conduction was evident. In addition, when ventricular conductivity increased, anterograde conduction became established. These findings suggest that source-sink mismatch was in play[10,16].
A thin AP may be unable to deliver the current required for propagation of excitation. As ventricular conductivity increases, electrotonic current rises in the ventricular wall; even a thin AP then provides a current adequate in terms of excitation propagation. As AP conductivity increases, anterograde conduction is blocked; an electrotonic effect may be in play[17]. In the monodomain model, many myocardial units interact electrically. The extent of such interactions depends on both the potential difference and the extent of intercellular conductivity. As that conductivity increases, the electrotonic current rises in both the waveback area and the wavefront. Therefore, the high-conductivity AP may be unable to deliver the electric current required for excitation propagation.

The Kent bundle AP is congenital in nature; however, symptomatic WPW in patients with normal hearts is agedependent[18]. We found that reduced AP conductivity triggered anterograde conduction. Our simulation may reflect symptom onset in patients with WPW syndrome.

We did not consider ion channel conductance. Furthermore, we did not explore retrograde conduction. Gallagher et al. showed that the AP refractory period differed markedly when conduction was anterograde or retrograde[2]. In addition, excitability depends on a mathematical formulation that reproduces membrane kinetics.

\section{Conclusion}

We used a theoretical approach to derive relationships among AP bundle size, myocardial conductivity, and anterograde conduction; we employed a simplified wall model. We offer new insights into the morphological and electrophysiological details of the AP.

\section{Acknowledgments}

This work was supported by JSPS KAKENHI Grant Numbers JP 17K01366, 16K09431.

\section{References}

[1] Qiu M, Lv B, Lin W, Ma J, Dong H. Sudden cardiac death due to the Wolff-Parkinson-White syndrome. Medicine dec 2018;97(51):e13248.

[2] Gallagher JJ, Pritchett EL, Sealy WC, Kasell J, Wallace AG. The preexcitation syndromes. Progress in cardiovascular diseases 1978;20(4):285-327.

[3] Arruda MS, McClelland JH, Wang X, Beckman KJ, Widman LE, Gonzalez MD, Nakagawa H, Lazzara R, Jackman WM. Development and validation of an ECG algorithm for identifying accessory pathway ablation site in WolffParkinson-White syndrome. Journal of cardiovascular electrophysiology jan 1998;9(1):2-12.

[4] Boersma L, García-Moran E, Mont L, Brugada J. Accessory pathway localization by QRS polarity in children with 
Wolff-Parkinson-White syndrome. Journal of cardiovascular electrophysiology dec 2002;13(12):1222-6.

[5] Nakagawa H, Jackman WM. Catheter ablation of paroxysmal supraventricular tachycardia. Circulation nov 2007; 116(21):2465-2478.

[6] Matsuyama T, Haraguchi R, Nakashima J, Kusano K, Ishibashi-Ueda $\mathrm{H}$. Three-dimensional histologic reconstruction of remnant functional accessory atrioventricular myocardial connections in a case of Wolff-Parkinson-White syndrome. Cardiovascular Pathology nov 2018;37:1-4.

[7] Wei D, Aoki M, Okamoto Y, Musha T, Harumi K. Computer simulation of the Wolff-Parkinson-White syndrome utilizing a human heart model. Japanese Heart Journal 1987;28(5):707-718.

[8] Debbarma J, Das P, Debbarma A, Chakraborty PN. Myocardial bridging and sudden death: a care report. Journal of Evolution of Medical and Dental Sciences jan 2015; 4(01):116-119.

[9] LaRocca TJ, Beyersdorf GB, Li W, Foltz R, Patel AR, Tanel RE. Comparison of electrophysiologic profiles in pediatric patients with incidentally identified pre-excitation compared with Wolff-Parkinson-White syndrome. The American Journal of Cardiology aug 2019;124(3):389-395.

[10] Kléber AG, Rudy Y. Basic mechanisms of cardiac impulse propagation and associated arrhythmias. Physiological Reviews apr 2004;84(2):431-488.

[11] Courtemanche M, Ramirez RJ, Nattel S. Ionic mechanisms underlying human atrial action potential properties: insights from a mathematical model. American Journal of Physiology Heart and Circulatory Physiology jul 1998; 275(1):H301-H321.

[12] O’Hara T, Virág L, Varró A, Rudy Y. Simulation of the undiseased human cardiac ventricular action potential: model formulation and experimental validation. PLoS Computational Biology may 2011;7(5):e1002061.

[13] Ashihara T, Suzuki T, Namba T, Inagaki M, Ikeda T, Ito M,
Kinoshita M, Nakazawa K. Simulated electrocardiogram of spiral wave reentry in a mathematical ventricular model. In Yamaguchi T (ed.), Clinical Application of Computational Mechanics to the Cardiovascular System. Tokyo: Springer Japan, 2000; 205-216.

[14] Kadish A, Shinnar M, Moore EN, Levine JH, Balke CW, Spear JF. Interaction of fiber orientation and direction of impulse propagation with anatomic barriers in anisotropic canine myocardium. Circulation dec 1988;78(6):14781494.

[15] Saffitz J, Yamada K. Gap junction distribution in the heart. In Zipes D, Jalife J (eds.), Cardiac Electrophysiology: From cell to bedside, 3rd edition. WB Saunders, 2000; 179-187.

[16] Kinoshita Si, Iwamoto M, Tateishi K, Suematsu NJ, Ueyama D. Mechanism of spiral formation in heterogeneous discretized excitable media. Physical Review E jun 2013;87(6):062815.

[17] Joyner RW. Effects of the discrete pattern of electrical coupling on propagation through an electrical syncytium. Circulation Research feb 1982;50(2):192-200.

[18] Cohen MI, Triedman JK, Cannon BC, Davis AM, Drago F, Janousek J, Klein GJ, Law IH, Morady FJ, Paul T, Perry JC, Sanatani S, Tanel RE. PACES/HRS expert consensus statement on the management of the asymptomatic young patient with a Wolff-Parkinson-White (WPW, ventricular preexcitation) electrocardiographic pattern. Heart Rhythm jun 2012;9(6):1006-1024.

Address for correspondence:

Ryo Haraguchi

Graduate School of Applied Informatics, University of Hyogo 7-1-28 Minatojima-minamimachi, Chuo-ku, Kobe, Hyogo 6500047, Japan

haraguch@ai.u-hyogo.ac.jp 